\title{
A NOTE ON QUASI BI-SLANT SUBMANIFOLDS OF COSYMPLECTIC MANIFOLDS
}

\author{
Mehmet Akif AKYOL ${ }^{1}$ and Selahattin BEYENDI ${ }^{2}$ \\ ${ }^{1}$ Department of Mathematics, Faculty of Arts and Sciences, Bingol University,12000, Bingöl, \\ TURKEY \\ ${ }^{2}$ Faculty of Education, Inonu University, 44000, Malatya, TURKEY
}

\begin{abstract}
The aim of the present paper is to define and study the notion of quasi bi-slant submanifolds of almost contact metric manifolds. We mainly concerned with quasi bi-slant submanifolds of cosymplectic manifolds as a generalization of slant, semi-slant, hemi-slant, bi-slant and quasi hemi-slant submanifolds. First, we give non-trivial examples in order to demostrate the method presented in this paper is effective and investigate the geometry of distributions. Moreover, We study these types of submanifolds with parallel canonical structures.
\end{abstract}

\section{INTRODUCTION}

Study of submanifolds theory has shown an increasing development in image processing, computer design, economic modeling as well as in mathematical physics and in mechanics. In this manner, B-Y. Chen [6] initiated the notion of slant submanifold as a generalization of both holomorphic (invariant) and totally real submanifold (anti-invariant) of an almost Hermitian manifold. Inspried by B-Y. Chen's paper, many geometers have studied this notion in the different kind of structures: (see 7], 22, [23]). Many consequent results on slant submanifolds are collected in his book 5. After this notion, as a generalization of semi-slant submanifold which was defined by N. Papaghiuc [19] (see also [8]). A. Carriazo 3] and 4 introduced the notion of bi-slant submanifold under the name anti-slant submanifold. However, B. Şahin called these submanifolds hemi-slant submanifolds in [21]. (See also [9] and [10, [20, 24]).

2020 Mathematics Subject Classification. 53C15, 53B20.

Keywords and phrases. Slant submanifold, bi-slant submanifold, quasi bi-slant submanifold, cosymplectic manifold.

$\square$ mehmetakifakyol@bingol.edu.tr-Corresponding author; selahattin.beyendi@inonu.edu.tr

(D) 0000-0003-2334-6955; 0000-0002-1037-6410.

(C)2020 Ankara University Communications Faculty of Sciences University of Ankara-Series A1 Mathematics and Statistics 
Furthermore, the submanifolds of a cosymplectic manifold have been studied by many geometers: See [11], [12], [13], [14], 15], [16], 18]. Taking into account of the above studies, we are motivated to fill a gap in the literature by giving the notion of quasi bi-slant submanifolds in which the tangent bundle consist of one invariant and two slant distributions and the Reeb vector field. In this paper, as a generalization of slant, semi-slant, hemi-slant, bi-slant and quasi hemi-slant submanifolds, we introduce quasi bi-slant submanifolds and investigate the geometry of distributions in detail.

The paper is organized as follows: In section 2, we recall basic formulas and definitions for a cosymplectic manifold and their submanifolds. In section 3, we introduce the notion of quasi bi-slant submanifolds, giving a non-tirivial example and obtain some basic results for the next sections. In section 4, we give some necessary and sufficient conditions for the geometry of distributions. Finally, we study these types of submanifolds with parallel canonical structures.

\section{Preliminaries}

In this section, we give the definition of cosymplectic manifold and some background on submanifolds theory.

A $(2 m+1)$-dimensional $C^{\infty}$-manifold $M$ said to have an almost contact structure if there exist on $M$ a tensor field $\varphi$ of type (1,1), a vector field $\xi$ and 1-form $\eta$ satisfying:

$$
\varphi^{2}=-I+\eta \otimes \xi, \quad \varphi \xi=0, \quad \eta \circ \varphi=0, \quad \eta(\xi)=1
$$

There always exists a Riemannian metric $g$ on an almost contact manifold $M$ satisfying the following conditions

$$
g(\varphi X, \varphi Y)=g(X, Y)-\eta(X) \eta(Y), \quad \eta(X)=g(X, \xi)
$$

where $X, Y$ are vector fields on $M$.

An almost contact structure $(\varphi, \xi, \eta)$ is said to be normal if the almost complex structure $J$ on the product manifold $M \times \mathbb{R}$ is given by

$$
J\left(X, f \frac{d}{d t}\right)=\left(\varphi X-f \xi, \eta(X) \frac{d}{d t}\right)
$$

where $f$ is a $C^{\infty}$-function on $M \times \mathbb{R}$ has no torsion i.e., $J$ is integrable. The condition for normality in terms of $\varphi, \xi$ and $\eta$ is $[\varphi, \varphi]+2 d \eta \otimes \xi=0$ on $M$, where $[\varphi, \varphi]$ is the Nijenhuis tensor of $\varphi$. Finally, the fundamental two-form $\Phi$ is defined $\Phi(X, Y)=g(X, \varphi Y)$.

An almost contact metric structure $(\varphi, \xi, \eta, g)$ is said to be cosymplectic, if it is normal and both $\Phi$ and $\eta$ are closed ( [1], [2], [16]), and the structure equation of a cosymplectic manifold is given by

$$
\left(\nabla_{X} \varphi\right) Y=0
$$


for any $X, Y$ tangent to $M$, where $\nabla$ denotes the Riemannian connection of the metric $g$ on $M$. Moreover, for cosymplectic manifold

$$
\nabla_{X} \xi=0 .
$$

Example. ( [17]) $\mathbb{R}^{2 n+1}$ with Cartesian coordinates $\left(x_{i}, y_{i}, z\right)(i=1, \ldots, n)$ and its usual contact form

$$
\eta=d z \text { and } \xi=\frac{\partial}{\partial z}
$$

here $\xi$ is the characteristic vector field and its Riemannian metric $g$ and tensor field $\varphi$ are given by

$$
g=\sum_{i=1}^{n}\left(\left(d x_{i}\right)^{2}+\left(d y_{i}\right)^{2}\right)+(d z)^{2}, \quad \varphi=\left(\begin{array}{ccc}
0 & \delta_{i j} & 0 \\
-\delta_{i j} & 0 & 0 \\
0 & 0 & 0
\end{array}\right), \quad i=1, \ldots, n .
$$

This gives a cosymplectic manifold on $\mathbb{R}^{2 n+1}$. The vector fields $e_{i}=\frac{\partial}{\partial y_{i}}, e_{n+i}=\frac{\partial}{\partial x_{i}}$, $\xi$ form a $\varphi$-basis for the cosymplectic structure. On the other hand, it can be shown that $\mathbb{R}^{2 n+1}(\varphi, \xi, \eta, g)$ is a cosymplectic manifold.

Let $M$ be a Riemannian manifold isometrically immersed in $\bar{M}$ and induced Riemannian metric on $M$ is denoted by the same symbol $g$ throughout this paper. Let $\mathcal{A}$ and $h$ denote the shape operator and second fundamental form, respectively, of immersion of $M$ into $\bar{M}$. The Gauss and Weingarten formulas of $M$ into $\bar{M}$ are given by $[6]$

$$
\bar{\nabla}_{X} Y=\nabla_{X} Y+h(X, Y)
$$

and

$$
\bar{\nabla}_{X} V=-\mathcal{A}_{V} X+\nabla_{X}^{\perp} V
$$

for any vector fields $X, Y \in \Gamma(T M)$ and $V \in \Gamma\left(T^{\perp} M\right)$, where $\nabla$ is the induced connection on $M$ and $\nabla^{\perp}$ represents the connection on the normal bundle $T^{\perp} M$ of $M$ and $A_{V}$ is the shape operator of $M$ with respect to normal vector $V \in \Gamma\left(T^{\perp} M\right)$. Moreover, $\mathcal{A}_{V}$ and $h$ are related by

$$
g(h(X, Y), V)=g\left(\mathcal{A}_{V} X, Y\right)
$$

for any vector fields $X, Y \in \Gamma(T M)$ and $V \in \Gamma\left(T^{\perp} M\right)$.

If $h(X, Y)=0$ for all $X, Y \in \Gamma(T M)$, then $M$ is said to be totally geodesic.

\section{QUASI BI-SLANT SUBMANIFOLDS OF COSMYPLECTIC MANIFOLDS}

In this section, we define the concept of quasi bi-slant submanifolds of cosymplectic manifolds, giving a non-trivial example and obtain some related results for later use.

Definition 3.1. A submanifold $M$ of cosymplectic manifold $(\bar{M}, \varphi, \xi, \eta, \bar{g})$ is called quasi bi-slant if there exists four orthogonal distributions $\mathcal{D}, \mathcal{D}_{1}$ and $\mathcal{D}_{2}$ and $\xi$ of $M$, at the point $p \in M$ such that 
(i) $T M=\mathcal{D} \oplus \mathcal{D}_{1} \oplus \mathcal{D}_{2} \oplus\langle\xi\rangle$

(ii) The distribution $\mathcal{D}$ is invariant, i.e. $\varphi \mathcal{D}=\mathcal{D}$.

(iii) $\varphi \mathcal{D}_{1} \perp \mathcal{D}_{2}$ and $\varphi \mathcal{D}_{2} \perp \mathcal{D}_{1}$;

(iv) The distributions $\mathcal{D}_{1}, \mathcal{D}_{2}$ are slant with slant angle $\theta_{1}, \theta_{2}$, respectively.

Taking the dimension of distributions $\mathcal{D}, \mathcal{D}_{1}$ and $\mathcal{D}_{2}$ are $m_{1}, m_{2}$ and $m_{3}$, respectively. One can easily see the following cases:

- If $m_{1} \neq 0$ and $m_{2}=m_{3}=0$, then $M$ is a invariant submanifold.

- If $m_{1}=m_{2}=0$ and $\theta_{2}=\frac{\pi}{2}$ then $M$ is an anti-invariant submanifold.

- If $m_{1}=0, m_{2} \neq m_{3} \neq 0, \theta_{1}=0$ and $\theta_{2}=\frac{\pi}{2}$ then $M$ is a semi-invariant submanifold.

- If $m_{1}=m_{2}=0$ and $0<\theta_{2}<\frac{\pi}{2}$ then $M$ is a slant submanifold.

- If $m_{1}=0, m_{2} \neq m_{3} \neq 0, \theta_{1}=0$ and $0<\theta_{2}<\frac{\pi}{2}$ then $M$ is a semi-slant submanifold.

- If $m_{1}=0, m_{2} \neq m_{3} \neq 0, \theta_{1}=\frac{\pi}{2}$ and $0<\theta_{2}<\frac{\pi}{2}$ then $M$ is a hemi-slant submanifold.

- If $m_{1}=0, m_{2} \neq m_{3} \neq 0$, and $\theta_{1}$ and $\theta_{2}$ are different from either 0 and $\frac{\pi}{2}$, then $M$ is a bi-slant submanifold.

If $m_{1} \neq m_{2} \neq m_{3} \neq 0$ and $\theta_{1}, \theta_{2} \neq 0, \frac{\pi}{2}$, then $M$ is called a proper quasi bi-slant submanifold.

Remark 3.2. In this paper, we assume that $M$ is proper quasi bi-slant submanifold of a cosymplectic manifold $\bar{M}$.

Now, we present an example of proper quasi bi-slant submanifold in $\mathbb{R}^{11}$.

Example. We will use the canonical contact structure $\varphi$ defined by

$$
\varphi\left(x_{1}, y_{1}, \ldots, x_{n}, y_{n}, z\right)=\left(y_{1},-x_{1}, \ldots, y_{n},-x_{n}, 0\right) .
$$

Thus we have $\varphi\left(\partial x_{i}\right)=\partial y_{i}, \varphi\left(\partial y_{j}\right)=-\partial x_{j}$ and $\varphi(\partial z)=0, \quad 1 \leq i, j \leq 5$ where $\partial x_{i}=\frac{\partial}{\partial x_{i}}$. For any pair of real numbers $\theta_{1}, \theta_{2}$ satisfying $0<\theta_{1}, \theta_{2}<\frac{\pi}{2}$, let us consider submanifold $M_{\theta_{1}, \theta_{2}}$ of $\mathbb{R}^{11}$ defined by

$\pi_{\theta_{1}, \theta_{2}}(u, s, w, k, t, r, z)=\left(u, s \cos \theta_{1}, 0, s \sin \theta_{1}, \omega, k \cos \theta_{2}, 0, k \sin \theta_{2}, t, r, z\right)$. If we take

$$
\begin{gathered}
e_{1}=\partial x_{1}, \quad e_{2}=\cos \theta_{1} \partial y_{1}+\sin \theta_{1} \partial y_{2}, \\
e_{3}=\partial x_{3}, \quad e_{4}=\cos \theta_{2} \partial y_{3}+\sin \theta_{2} \partial y_{4}, \\
e_{5}=\partial x_{5}, \quad e_{6}=\partial y_{5}, \quad e_{7}=\xi=\partial z
\end{gathered}
$$

then the restriction of $e_{1}, \ldots, e_{7}$ to $M$ forms an orthonormal frame of the tangent bundle $T M$. Obviously, we get

$$
\begin{gathered}
\varphi e_{1}=\partial y_{1}, \varphi e_{2}=-\cos \theta_{1} \partial x_{1}-\sin \theta_{1} \partial x_{2}, \varphi e_{3}=\partial y_{3} \\
\varphi e_{4}=-\cos \theta_{2} \partial x_{3}-\sin \theta_{2} \partial x_{4}, \varphi e_{5}=\partial y_{5}, \varphi e_{6}=-\partial x_{5} .
\end{gathered}
$$

Let us put $\mathcal{D}_{1}=\operatorname{Span}\left\{e_{1}, e_{2}\right\}, \mathcal{D}_{2}=\operatorname{Span}\left\{e_{3}, e_{4}\right\}$, and $\mathcal{D}=\operatorname{Span}\left\{e_{5}, e_{6}\right\}$. Then obviously $\mathcal{D}_{1}, \mathcal{D}_{2}$ and $\mathcal{D}$, satisfy the definition of quasi bi-slant submanifold $M_{\theta_{1}, \theta_{2}}$ 
defined by $\pi_{\theta_{1}, \theta_{2}}$ is a proper quasi bi-slant submanifold of $\mathbb{R}^{11}$ with $\theta_{1}, \theta_{2}$ as its bi-slant angles.

Let $M$ be a quasi bi-slant submanifold of a cosymplectic manifold $\bar{M}$. Then, for any $X \in \Gamma(T M)$, we have

$$
X=\mathcal{P} X+\mathcal{Q} X+\mathcal{R} X+\eta(X) \xi
$$

where $\mathcal{P}, \mathcal{Q}$ and $\mathcal{R}$ denote the projections on the distributions $\mathcal{D}, \mathcal{D}_{1}$ and $\mathcal{D}_{2}$, recpectively.

$$
\varphi X=\mathcal{T} X+\mathcal{F} X
$$

where $\mathcal{T} X$ and $\mathcal{F} X$ are tangential and normal components on $M$. Making now use of (3.1) and 3.2), we get immediately

$$
\varphi X=\mathcal{T} \mathcal{P} X+\mathcal{T} \mathcal{Q} X+\mathcal{F} \mathcal{Q} X+\mathcal{T} \mathcal{R} X+\mathcal{F} \mathcal{R} X
$$

here since $\varphi \mathcal{D}=\mathcal{D}$, we have $\mathcal{F P} X=0$. Thus we get

$$
\varphi(T M)=\mathcal{D} \oplus \mathcal{T D}_{1} \oplus \mathcal{T} \mathcal{D}_{2}
$$

and

$$
T^{\perp} M=\mathcal{F D}_{1} \oplus \mathcal{F D}_{2} \oplus \mu
$$

where $\mu$ is the orthogonal complement of $\mathcal{F} \mathcal{D}_{1} \oplus \mathcal{F D}_{2}$ in $T^{\perp} M$ and it is invariant with recpect to $\varphi$. Also, for any $Z \in T^{\perp} M$, we have

$$
\varphi Z=\mathcal{B} Z+\mathcal{C} Z
$$

where $\mathcal{B} Z \in \Gamma(T M)$ and $\mathcal{C} Z \in \Gamma\left(T^{\perp} M\right)$.

Taking into account of the condition (iii) in Definition (3.1), (3.2) and (3.6), we obtain the followings:

Lemma 3.3. Let $M$ be a quasi bi-slant submanifold of a cosymplectic manifold $\bar{M}$. Then, we have
(a) $T \mathcal{D}_{1} \subset \mathcal{D}_{1}$,
(b) $T \mathcal{D}_{2} \subset \mathcal{D}_{2}$,
(c) $\mathcal{B} \mathcal{F} \mathcal{D}_{1}=\mathcal{D}_{1}$
(d) $\mathcal{B} \mathcal{F} \mathcal{D}_{2}=\mathcal{D}_{2}$.

With the help of 3.2 and (3.6), we obtain the following Lemma.

Lemma 3.4. Let $M$ be a quasi bi-slant submanifold of a cosymplectic manifold $\bar{M}$. Then, we have

$$
\begin{aligned}
& \text { (a) } \mathcal{T}^{2} U_{1}=-\cos ^{2} \theta_{1} U_{1}, \quad \text { (b) } \mathcal{T}^{2} U_{2}=-\cos ^{2} \theta_{2} U_{2}, \\
& \text { (c) } \mathcal{B F} U_{1}=-\sin ^{2} \theta_{1} U_{1}, \quad \text { (d) } \mathcal{B F} U_{2}=-\sin ^{2} \theta_{1} U_{2} \text {, } \\
& \text { (e) } \mathcal{T}^{2} U_{1}+\mathcal{B F} U_{1}=-U_{1}, \quad \text { (f) } \mathcal{T}^{2} U_{2}+\mathcal{B F} U_{2}=-U_{2} \text {, }
\end{aligned}
$$

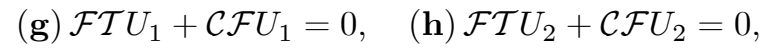

for any $U_{1} \in \mathcal{D}_{1}$ and $U_{2} \in \mathcal{D}_{2}$.

By using (2.3), Definition (3.1), (3.2) and (3.6), we obtain the following Lemma.

Lemma 3.5. Let $M$ be a quasi bi-slant submanifold of a cosymplectic manifold $\bar{M}$. Then, we have 
(i) $\mathcal{T}_{i}^{2} U_{i}=-\cos ^{2} \theta_{i} U_{i}$

(ii) $g\left(\mathcal{T}_{i} U_{i}, \mathcal{T}_{i} V_{i}\right)=\left(\cos ^{2} \theta_{i}\right) g\left(U_{i}, V_{i}\right)$,

(iii) $g\left(\mathcal{F}_{i} U_{i}, \mathcal{F}_{i} V_{i}\right)=\left(\sin ^{2} \theta_{i}\right) g\left(U_{i}, V_{i}\right)$

for any $i=1,2, U_{1}, V_{1} \in \Gamma\left(\mathcal{D}_{1}\right)$ and $U_{2}, V_{2} \in \Gamma\left(\mathcal{D}_{2}\right)$.

We need the following lemma for later use.

Lemma 3.6. Let $M$ be a quasi bi-slant submanifold of a cosymplectic manifold $\bar{M}$, then for any $Z_{1}, Z_{2} \in \Gamma(T M)$, we have the following

$$
\nabla_{Z_{1}} \mathcal{T} Z_{2}-\mathcal{T} \nabla_{Z_{1}} Z_{2}=\mathcal{A}_{\mathcal{F} Z_{2}} Z_{1}+\mathcal{B} h\left(Z_{1}, Z_{2}\right)
$$

and

$$
\nabla_{Z_{1}}^{\perp} \mathcal{F} Z_{2}-\mathcal{F} \nabla_{Z_{1}} Z_{2}=\mathcal{C} h\left(Z_{1}, Z_{2}\right)-h\left(Z_{1}, \mathcal{T} Z_{2}\right)
$$

Proof. Since $\bar{M}$ is a cosmyplectic manifold, we have that

$$
\left(\bar{\nabla}_{Z_{1}} \varphi\right) Z_{2}=0
$$

which implies that

$$
\bar{\nabla}_{Z_{1}} \varphi Z_{2}-\varphi \bar{\nabla}_{Z_{1}} Z_{2}=0 .
$$

By using 2.5 and (3.2), we get

$$
\bar{\nabla}_{Z_{1}} \mathcal{T} Z_{2}+\bar{\nabla}_{Z_{1}} \mathcal{F} Z_{2}-\varphi\left(\nabla_{Z_{1}} Z_{2}+h\left(Z_{1}, Z_{2}\right)\right)=0 .
$$

Taking into account of (2.5), (2.6), (3.2) and $(3.6)$, we obtain

$$
\begin{aligned}
& \nabla_{Z_{1}} \mathcal{T} Z_{2}+h\left(Z_{1}, \mathcal{T} Z_{2}\right)-\mathcal{A}_{\mathcal{F} Z_{2}} Z_{1}+\nabla_{Z_{1}}^{\perp} \mathcal{F} Z_{2} \\
& -\mathcal{T} \nabla_{Z_{1}} Z_{2}-\mathcal{F} \nabla_{Z_{1}} Z_{2}-\mathcal{B} h Z_{1}, Z_{2}-\mathcal{C} h\left(Z_{1}, Z_{2}\right)=0 .
\end{aligned}
$$

Comparing the tangential and normal components, we have the required results.

In a similar way, we have:

Lemma 3.7. Let $M$ be a quasi bi-slant submanifold of a cosymplectic manifold $\bar{M}$, then we have the following

$$
\nabla_{Z_{1}} \mathcal{B} W_{1}-\mathcal{B} \nabla_{Z_{1}}^{\perp} W_{1}=\mathcal{A}_{\mathcal{C} W_{1}} Z_{1}-\mathcal{T} \mathcal{A}_{W_{1}} Z_{1}
$$

and

$$
\nabla_{Z_{1}}^{\perp} \mathcal{C} W_{1}-\mathcal{C} \nabla_{Z_{1}}^{\perp} W_{1}=-\mathcal{F} \mathcal{A}_{W_{1}} Z_{1}-h\left(Z_{1}, \mathcal{B} W_{1}\right)
$$

for any $Z_{1} \in \Gamma(T M)$ and $W_{1} \in \Gamma\left(T^{\perp} M\right)$. 


\section{INTEGRABILITY AND TOTALLY GEODESIC FOLIATIONS}

In this section we give some necessary and sufficient conditions for the integrability of the distributions.

First, we have the following theorem:

Theorem 4.1. Let $M$ be a quasi bi-slant submanifold of $\bar{M}$. The invariant distribution $\mathcal{D}$ is integrable if and only if

$$
g\left(\mathcal{T}\left(\nabla_{X} \mathcal{T} Y-\nabla_{Y} \mathcal{T} X\right), Z\right)=g(h(X, \mathcal{T} Y)-h(Y, \mathcal{T} X), \varphi Q Z+\varphi R Z)
$$

for any $X, Y \in \Gamma(\mathcal{D})$ and $Z \in \Gamma\left(\mathcal{D}_{1} \oplus \mathcal{D}_{2}\right)$.

Proof. The distribution $\mathcal{D}$ is integrable on $M$ if and only if

$$
g([X, Y], \xi)=0 \quad \text { and } \quad g([X, Y], Z)=0
$$

for any $X, Y \in \Gamma(\mathcal{D}), Z \in \Gamma\left(\mathcal{D}_{1} \oplus \mathcal{D}_{2}\right)$ and $\xi \in \Gamma(T M)$. Since $M$ is a cosymplectic manifold, we immediately have $g([X, Y], \xi)=0$. Thus $\mathcal{D}$ is integrable if and only if $g([X, Y], Z)=0$. Now, for any $X, Y \in \mathcal{D}$ and $Z=\mathcal{Q} Z+\mathcal{R} Z \in \Gamma\left(\mathcal{D}_{1} \oplus \mathcal{D}_{2}\right)$, by using 2.2, 2.5, we obtain

$$
g([X, Y], Z)=g\left(\varphi \bar{\nabla}_{X} Y, \varphi Z\right)-\eta\left(\bar{\nabla}_{X} Y\right) \eta(Z)-g\left(\varphi \bar{\nabla}_{Y} X, \varphi Z\right)+\eta\left(\bar{\nabla}_{Y} X\right) \eta(Z) .
$$

Now, using 2.4, 3.2 and $\mathcal{F} Y=0$ for any $Y \in \Gamma(\mathcal{D})$, we have

$$
\begin{aligned}
g([X, Y], Z) & =g\left(\bar{\nabla}_{X} \varphi Y, \varphi Z\right)-g\left(\bar{\nabla}_{Y} \varphi X, \varphi Z\right) \\
& =g\left(\bar{\nabla}_{X} \mathcal{T} Y, \varphi Z\right)-g\left(\bar{\nabla}_{Y} \mathcal{T} X, \varphi Z\right) .
\end{aligned}
$$

Taking into account of 2.5 and $\sqrt{3.3}$ in the above equation, we get

$$
\begin{aligned}
g([X, Y], Z) & =-g\left(\varphi \nabla_{X} \mathcal{T} Y, Z\right)+g(h(X, \mathcal{T} Y), \varphi Z) \\
& +g\left(\varphi \nabla_{Y} \mathcal{T} X, Z\right)-g(h(Y, \mathcal{T} X), \varphi Z)
\end{aligned}
$$

Now again taking into account the equation 3.2 , we obtain

$$
\begin{aligned}
g([X, Y], Z) & =g\left(\mathcal{T}\left(\nabla_{Y} \mathcal{T} X-\nabla_{X} \mathcal{T} Y\right), Z\right) \\
& +g(h(X, \mathcal{T} Y)-h(Y, \mathcal{T} X), \varphi Q Z+\varphi R Z)
\end{aligned}
$$

which completes the proof.

For the slant distribution $\mathcal{D}_{1}$, we have:

Theorem 4.2. Let $M$ be a quasi bi-slant submanifold of $\bar{M}$. The slant distribution $\mathcal{D}_{1}$ is integrable if and only if

$$
\begin{aligned}
g\left(\nabla_{U_{1}}^{\perp} \mathcal{F} V_{1}+\nabla_{V_{1}}^{\perp} \mathcal{F} U_{1}, \mathcal{F} \mathcal{R} Z\right) & =g\left(\mathcal{A}_{\mathcal{F} \mathcal{T} V_{1}} U_{1}-\mathcal{A}_{\mathcal{F} \mathcal{T} U_{1}} V_{1}, Z\right) \\
& +g\left(\mathcal{A}_{\mathcal{F} V_{1}} U_{1}+\mathcal{A}_{\mathcal{F} U_{1}} V_{1}, \mathcal{T} Z\right)
\end{aligned}
$$

for any $U_{1}, V_{1} \in \Gamma\left(\mathcal{D}_{1}\right), Z \in \Gamma\left(\mathcal{D} \oplus \mathcal{D}_{2}\right)$. 
A NOTE ON QUASI BI-SLANT SUBMANIFOLDS OF COSYMPLECTIC MANIFOLDS 1515

Proof. The distribution $\mathcal{D}_{1}$ is integrable on $M$ if and only if

$$
g\left(\left[U_{1}, V_{1}\right], \xi\right)=0 \quad \text { and } \quad g\left(\left[U_{2}, V_{2}\right], Z\right)=0
$$

for any $U_{1}, V_{1} \in \Gamma\left(\mathcal{D}_{1}\right), Z \in \Gamma\left(\mathcal{D} \oplus \mathcal{D}_{2}\right)$ and $\xi \in \Gamma(T M)$. The first case is trivial. Thus $\mathcal{D}_{1}$ is integrable if and only if $g\left(\left[U_{1}, V_{1}\right], Z\right)=0$. Now, for any $U_{1}, V_{1} \in \mathcal{D}_{1}$ and $Z=\mathcal{P} Z+\mathcal{R} Z \in \Gamma\left(\mathcal{D} \oplus \mathcal{D}_{2}\right)$, by using 2.2 , 2.5, we obtain

$$
\begin{aligned}
g\left(\left[U_{1}, V_{1}\right], Z\right) & =-g\left(\bar{\nabla}_{U_{1}} \varphi \mathcal{T} V_{1}, Z\right)-g\left(\bar{\nabla}_{U_{1}} \mathcal{F} V_{1}, \varphi Z\right) \\
& +g\left(\bar{\nabla}_{V_{1}} \varphi \mathcal{T} U_{1}, Z\right)-g\left(\bar{\nabla}_{V_{1}} \mathcal{F} U_{1}, \varphi Z\right) .
\end{aligned}
$$

Taking into account the equation lemma (3.5) (i) in the above equation, we get

$$
\begin{aligned}
g\left(\left[U_{1}, V_{1}\right], Z\right) & =\cos ^{2} \theta_{1} g\left(\left[U_{1}, V_{1}\right], Z\right)-g\left(\bar{\nabla}_{U_{1}} \mathcal{F} \mathcal{T} V_{1}-\bar{\nabla}_{V_{1}} \mathcal{F} \mathcal{T} U_{1}, Z\right) \\
& -g\left(\bar{\nabla}_{U_{1}} \mathcal{F} V_{1}+\bar{\nabla}_{V_{1}} \mathcal{F} U_{1}, \varphi \mathcal{P} Z+\varphi \mathcal{R} Z\right) .
\end{aligned}
$$

Now, using (2.6) and (3.3), we obtain

$$
\begin{aligned}
g\left(\left[U_{1}, V_{1}\right], Z\right) & =\cos ^{2} \theta_{1} g\left(\left[U_{1}, V_{1}\right], Z\right)+g\left(\mathcal{A}_{\mathcal{F} \mathcal{T} V_{1}} U_{1}-\mathcal{A}_{\mathcal{F} \mathcal{T} U_{1}} V_{1}, Z\right) \\
& +g\left(\mathcal{A}_{\mathcal{F} V_{1}} U_{1}+\mathcal{A}_{\mathcal{F} U_{1}} V_{1}, \mathcal{T} Z\right) \\
& -g\left(\nabla_{U_{1}}^{\perp} \mathcal{F} V_{1}+\nabla_{V_{1}}^{\perp} \mathcal{F} U_{1}, \mathcal{F} \mathcal{R} Z\right)
\end{aligned}
$$

or

$$
\begin{aligned}
\sin ^{2} \theta_{1} g\left(\left[U_{1}, V_{1}\right], Z\right) & =g\left(\mathcal{A}_{\mathcal{F} \mathcal{T} V_{1}} U_{1}-\mathcal{A}_{\mathcal{F} \mathcal{T} U_{1}} V_{1}, Z\right) \\
& +g\left(\mathcal{A}_{\mathcal{F} V_{1}} U_{1}+\mathcal{A}_{\mathcal{F} U_{1}} V_{1}, \mathcal{T} Z\right) \\
& -g\left(\nabla_{U_{1}}^{\perp} \mathcal{F} V_{1}+\nabla_{V_{1}}^{\perp} \mathcal{F} U_{1}, \mathcal{F} \mathcal{R} Z\right)
\end{aligned}
$$

which gives the assertion.

In a similar way, we obtain the following case for the slant distribution $\mathcal{D}_{2}$.

Theorem 4.3. Let $M$ be a quasi bi-slant submanifold of $\bar{M}$. The slant distribution $\mathcal{D}_{2}$ is integrable if and only if

$$
\begin{aligned}
\mathcal{T}\left(\nabla_{U_{2}} \mathcal{T} V_{2}-\mathcal{A}_{\mathcal{F} V_{2}} U_{2}\right) & \in \Gamma\left(\mathcal{D}_{2}\right), \\
\mathcal{B}\left(h\left(U_{2}, T V_{2}\right)+\nabla_{U_{2}}^{\perp} \mathcal{F} V_{2}\right) & \in \Gamma\left(T^{\perp} M\right)
\end{aligned}
$$

and

$$
g\left(\mathcal{A}_{\mathcal{F} Z} V_{2}-\nabla_{V_{2}} \mathcal{T} Z, \mathcal{T} U_{2}\right)=g\left(h\left(V_{2}, \mathcal{T} Z\right)+\nabla_{V_{2}}^{\perp} \mathcal{F} Z, \mathcal{F} U_{2}\right)
$$

for any $U_{2}, V_{2} \in \Gamma\left(\mathcal{D}_{2}\right), Z=\mathcal{P} Z+\mathcal{Q} Z \in \Gamma\left(\mathcal{D} \oplus \mathcal{D}_{1}\right)$ and $W \in \Gamma\left(T^{\perp} M\right)$.

Theorem 4.4. Let $M$ be a quasi bi-slant submanifold of $\bar{M}$. The invariant distribution $\mathcal{D}$ defines totally geodesic foliation on $M$ if and only if

$$
g\left(\nabla_{X} \mathcal{T} Y, \mathcal{T} Z\right)=-g(h(X, \mathcal{T} Y), \mathcal{F} Z)
$$

and

$$
\mathcal{F} \nabla_{X} \mathcal{T} Y_{1}+\mathcal{C} h(X, \mathcal{T} Y) \in \Gamma(T M)
$$

for any $X, Y \in \Gamma(\mathcal{D}), Z=\mathcal{Q} Z+\mathcal{R} Z \in \Gamma\left(\mathcal{D}_{1} \oplus \mathcal{D}_{2}\right)$ and $W \in \Gamma\left(T^{\perp} M\right)$. 
Proof. The distribution $\mathcal{D}$ defines a totaly geodesic foliation on $M$ if and only if $g\left(\bar{\nabla}_{X} Y, \xi\right)=0, g\left(\bar{\nabla}_{X} Y, Z\right)=0$ and $g\left(\bar{\nabla}_{X} Y, W\right)=0$ for any $X, Y \in \Gamma(\mathcal{D})$, $Z=\mathcal{Q} Z+\mathcal{R} Z \in \Gamma\left(\mathcal{D}_{1} \oplus \mathcal{D}_{2}\right)$ and $W \in \Gamma\left(T^{\perp} M\right)$. Then by using 2.2) and (2.4), we obtain

$$
g\left(\bar{\nabla}_{X} Y, \xi\right)=X g(Y, \xi)-g\left(Y, \bar{\nabla}_{X} \xi\right)=-g\left(Y, \bar{\nabla}_{X} \xi\right)=0 .
$$

On the other hand, using $(2.2)$, we find

$$
g\left(\bar{\nabla}_{X} Y, Z\right)=g\left(\bar{\nabla}_{X} \varphi Y, \varphi Z\right)=g\left(\bar{\nabla}_{X} \mathcal{T} Y, \varphi Z\right)
$$

here we have used $\mathcal{F} Y=0$ for any $Y \in \Gamma(\mathcal{D})$. Now, by using $(3.3)$ and $(2.5)$, we have

$$
\begin{aligned}
g\left(\bar{\nabla}_{X} Y, Z\right) & =g\left(\nabla_{X} \mathcal{T} Y+h(X, \mathcal{T} Y), \varphi \mathcal{Q} Z+\varphi \mathcal{R} Z\right) \\
& \left.=g\left(\nabla_{X} \mathcal{T} Y+h(X, \mathcal{T} Y), \mathcal{T} \mathcal{Q} Z+\mathcal{F} \mathcal{Q} Z+\mathcal{T} \mathcal{R} Z+\mathcal{F} \mathcal{R} Z\right)\right) \\
& \left.=g\left(\nabla_{X} \mathcal{T} Y, \mathcal{T} \mathcal{Q} Z+\mathcal{T} \mathcal{R} Z\right)+g(h(X, \mathcal{T} Y), \mathcal{F} \mathcal{Q} Z+\mathcal{F} \mathcal{R} Z)\right) \\
& =g\left(\nabla_{X} \mathcal{T} Y, \mathcal{T} Z\right)+g(h(X, \mathcal{T} Y), \mathcal{F} Z)
\end{aligned}
$$

for any $X, Y \in \Gamma(\mathcal{D})$ and $Z=\mathcal{Q} Z+\mathcal{R} Z \in \Gamma\left(\mathcal{D}_{1} \oplus \mathcal{D}_{2}\right)$. Now, for any $X, Y \in \Gamma(\mathcal{D})$ and $W \in \Gamma\left(T^{\perp} M\right)$, we have

$$
\begin{aligned}
g\left(\bar{\nabla}_{X} Y, W\right) & \left.=-g\left(\varphi \bar{\nabla}_{X_{1}} \varphi Y_{1}, W\right)=-g\left(\varphi\left(\nabla_{X} \mathcal{T} Y+h(X, \mathcal{T} Y)\right), W\right)\right) \\
& \left.=-g\left(\mathcal{T} \nabla_{X} \mathcal{T} Y+\mathcal{F} \nabla_{X} \mathcal{T} Y+\mathcal{B} h(X, \mathcal{T} Y)+\mathcal{C} h(X, \mathcal{T} Y), W\right)\right) \\
& =-g\left(\mathcal{F} \nabla_{X} \mathcal{T} Y+\mathcal{C} h(X, \mathcal{T} Y), W\right) .
\end{aligned}
$$

Thus proof follows 4.3 , (4.4) and 4.5).

Theorem 4.5. Let $M$ be a quasi bi-slant submanifold of $\bar{M}$. The slant distribution $\mathcal{D}_{1}$ defines totally geodesic foliation on $M$ if and only if

$$
\begin{aligned}
& g\left(\mathcal{A}_{\mathcal{F} \mathcal{T} V_{1}} U_{1}, Z\right)-g\left(\mathcal{A}_{\mathcal{F} V_{1}} U_{1}, \mathcal{T} \mathcal{P} Z\right) \\
& =g\left(\mathcal{A}_{\mathcal{F} V_{1}} U_{1}, \mathcal{T} \mathcal{R} Z\right)-g\left(\nabla_{U_{1}}^{\perp} \mathcal{F} V_{1}, \mathcal{F} \mathcal{R} Z\right)
\end{aligned}
$$

and

$$
\mathcal{F} \mathcal{A}_{\mathcal{F} V_{1}} U_{1}-\nabla_{U_{1}}^{\perp} \mathcal{F} \mathcal{T} V_{1}-\mathcal{C} \nabla_{U_{1}}^{\perp} \mathcal{F} V_{1} \in \Gamma(T M)
$$

for any $X, Y \in \Gamma(\mathcal{D}), Z=\mathcal{Q} Z+\mathcal{R} Z \in \Gamma\left(\mathcal{D}_{1} \oplus \mathcal{D}_{2}\right)$ and $W \in \Gamma\left(T^{\perp} M\right)$.

Proof. The distribution $\mathcal{D}_{1}$ defines a totaly geodesic foliation on $M$ if and only if $g\left(\bar{\nabla}_{U_{1}} V_{1}, \xi\right)=0, g\left(\bar{\nabla}_{U_{1}} V_{1}, Z\right)=0$ and $g\left(\bar{\nabla}_{U_{1}} V_{1}, W\right)=0$, for any $U_{1}, V_{1} \in \Gamma\left(\mathcal{D}_{1}\right)$, $Z=\mathcal{P} Z+\mathcal{R} Z \in \Gamma\left(\mathcal{D}_{1} \oplus \mathcal{D}_{2}\right)$ and $W \in \Gamma\left(T^{\perp} M\right)$. Since $M$ is a cosymplectic manifold, we immediately have $g\left(\bar{\nabla}_{U_{1}} V_{1}, \xi\right)=0$. Now, for any $U_{1}, V_{1} \in \Gamma\left(\mathcal{D}_{1}\right)$, and $Z=\mathcal{P} Z+\mathcal{R} Z \in \Gamma\left(\mathcal{D}_{1} \oplus \mathcal{D}_{2}\right)$, by using $(2.2)$ and $(2.4)$, we obtain

$$
g\left(\bar{\nabla}_{U_{1}} V_{1}, Z\right)=-g\left(\bar{\nabla}_{U_{1}} \varphi \mathcal{T} V_{1}, Z\right)+g\left(\bar{\nabla}_{U_{1}} \mathcal{F} V_{1}, \varphi \mathcal{P} Z+\varphi \mathcal{R} Z\right) .
$$

Now, by using lemma (3.5) (i), we get

$$
g\left(\bar{\nabla}_{U_{1}} V_{1}, Z\right)=\cos ^{2} \theta_{1} g\left(\bar{\nabla}_{U_{1}} V_{1}, Z\right)-g\left(-\mathcal{A}_{\mathcal{F} \mathcal{T} V_{1}} U_{1}+\nabla_{U_{1}}^{\perp} \mathcal{F} \mathcal{T} V_{1}, Z\right)
$$


A NOTE ON QUASI BI-SLANT SUBMANIFOLDS OF COSYMPLECTIC MANIFOLDS 1517

$$
\begin{aligned}
& +g\left(-\mathcal{A}_{\mathcal{F} V_{1}} U_{1}+\nabla_{U_{1}}^{\perp} \mathcal{F} V_{1}, \mathcal{T} \mathcal{P} Z\right) \\
& +g\left(-\mathcal{A}_{\mathcal{F} V_{1}} U_{1}+\nabla_{U_{1}}^{\perp} \mathcal{F} V_{1}, \mathcal{T} \mathcal{R} Z+\mathcal{F} \mathcal{R} Z\right)
\end{aligned}
$$

or

$$
\begin{aligned}
\sin ^{2} \theta_{1} g\left(\bar{\nabla}_{U_{1}} V_{1}, Z\right) & =g\left(\mathcal{A}_{\mathcal{F} \mathcal{T} V_{1}} U_{1}, Z\right)-g\left(\mathcal{A}_{\mathcal{F} V_{1}} U_{1}, \mathcal{T} \mathcal{P} Z\right) \\
& -g\left(\mathcal{A}_{\mathcal{F} V_{1}} U_{1}, \mathcal{T} \mathcal{R} Z\right)+g\left(\nabla_{U_{1}}^{\perp} \mathcal{F} V_{1}, \mathcal{F} \mathcal{R} Z\right) .
\end{aligned}
$$

Now, for any $U_{1}, V_{1} \in \Gamma(\mathcal{D})$ and $W \in \Gamma(T M)^{\perp}$, we have

$$
\begin{aligned}
g\left(\bar{\nabla}_{U_{1}} V_{1}, W\right) & =-g\left(\bar{\nabla}_{U_{1}} \varphi \mathcal{T} V_{1}, W\right)-g\left(\varphi\left(\bar{\nabla}_{U_{1}} \mathcal{F} V_{1}\right), W\right) \\
& -g\left(\varphi\left(-\mathcal{A}_{\mathcal{F} V_{1}} U_{1}+\nabla_{U_{1}}^{\perp} \mathcal{F} V_{1}\right), W\right) \\
& =\cos ^{2} \theta_{1} g\left(\bar{\nabla}_{U_{1}} V_{1}, W\right)-g\left(-\mathcal{A}_{\mathcal{F} \mathcal{T} V_{1}} U_{1}+\nabla_{U_{1}}^{\perp} \mathcal{F} \mathcal{T} V_{1}, W\right) \\
& -g\left(-\mathcal{T} \mathcal{A}_{\mathcal{F} V_{1}} U_{1}-\mathcal{F} \mathcal{A}_{\mathcal{F} V_{1}} U_{1}+\mathcal{B} \nabla_{U_{1}}^{\perp} \mathcal{F} V_{1}+\mathcal{C} \nabla_{U_{1}}^{\perp} \mathcal{F} V_{1}, W\right)
\end{aligned}
$$

or

$$
\begin{aligned}
\sin ^{2} \theta_{1} g\left(\bar{\nabla}_{U_{1}} V_{1}, W\right) & =-g\left(\nabla_{U_{1}}^{\perp} \mathcal{F} \mathcal{T} V_{1}, W\right)+g\left(\mathcal{F} \mathcal{A}_{\mathcal{F} V_{1}} U_{1}-\mathcal{C} \nabla_{U_{1}}^{\perp} \mathcal{F} V_{1}, W\right) \\
& =g\left(\mathcal{F} \mathcal{A}_{\mathcal{F} V_{1}} U_{1}-\nabla_{U_{1}}^{\perp} \mathcal{F} \mathcal{T} V_{1}-\mathcal{C} \nabla_{U_{1}}^{\perp} \mathcal{F} V_{1}, W\right) .
\end{aligned}
$$

Thus proof follows (4.8) and 4.9.

Theorem 4.6. Let $M$ be a quasi bi-slant submanifold of $\bar{M}$. The slant distribution $\mathcal{D}_{2}$ defines totally geodesic foliation on $M$ if and only if

$$
\begin{gathered}
\mathcal{T}\left(\nabla_{U_{2}} \mathcal{T} V_{2}-\mathcal{A}_{\mathcal{F} V_{2}} U_{2}\right) \in \Gamma\left(\mathcal{D}_{2}\right), \\
\mathcal{B}\left(h\left(U_{2}, T V_{2}\right)+\nabla_{U_{2}}^{\perp} \mathcal{F} V_{2}\right) \in \Gamma\left(T^{\perp} M\right)
\end{gathered}
$$

and

$$
g\left(\nabla_{U_{2}}^{\perp} \mathcal{F} \mathcal{T} V_{2}-\mathcal{F} \mathcal{A}_{V_{2}} U_{2}, W\right)=g\left(\nabla_{U_{2}}^{\perp} \mathcal{F} V_{2}, \mathcal{C} W\right)
$$

for any $U_{2}, V_{2} \in \Gamma\left(\mathcal{D}_{2}\right), Z=\mathcal{P} Z+\mathcal{Q} Z \in \Gamma\left(\mathcal{D} \oplus \mathcal{D}_{1}\right)$ and $W \in \Gamma\left(T^{\perp} M\right)$.

From theorem (4.4), 4.5) and (4.6), we have the following decomposition theorem:

Theorem 4.7. Let $M$ be a proper quasi bi-slant submanifold of a cosmyplectic manifold $\bar{M}$. Then $M$ is a local product Riemannian manifold of the form $M_{\mathcal{D}} \times$ $M_{\mathcal{D}_{1}} \times M_{\mathcal{D}_{2}}$, where $M_{\mathcal{D}}, M_{\mathcal{D}_{1}}$ and $M_{\mathcal{D}_{2}}$ are leaves of $\mathcal{D}, \mathcal{D}_{1}$ and $\mathcal{D}_{2}$, recpectively, if and only if the conditions (4.1), 4.2, 4.6), 4.7), 4.10, 4.11) and 4.12 hold. 


\section{QUASI BI-SLANT SUBMANIFOLDS WITH PARALLEL CANONICAL STRUCTURES}

In this section, we obtain some results for the quasi bi-slant submanifolds with parallel canonical structure. Let $M$ be a proper quasi bi-slant submanifold of a cosymplectic manifold $\bar{M}$. Then we define

$$
\begin{aligned}
& \left(\bar{\nabla}_{Z_{1}} \mathcal{T}\right) Z_{2}=\nabla_{Z_{1}} \mathcal{T} Z_{2}-\mathcal{T} \nabla_{Z_{1}} Z_{2} \\
& \left(\bar{\nabla}_{Z_{1}} \mathcal{F}\right) Z_{2}=\nabla_{Z_{1}}^{\perp} \mathcal{F} Z_{2}-\mathcal{F} \nabla_{Z_{1}} Z_{2} \\
& \left(\bar{\nabla}_{Z_{1}} \mathcal{B}\right) W_{1}=\nabla_{Z_{1}} \mathcal{B} W_{1}-\mathcal{B} \nabla_{Z_{1}}^{\perp} W_{1} \\
& \left(\bar{\nabla}_{Z_{1}} \mathcal{C}\right) W_{1}=\nabla_{Z_{1}}^{\perp} \mathcal{C} W_{1}-\mathcal{C} \nabla_{Z_{1}}^{\perp} W_{1}
\end{aligned}
$$

where $Z_{1}, Z_{2} \in \Gamma(T M)$ and $W_{1} \in \Gamma\left(T^{\perp} M\right)$.

Then, the endomorphism $\mathcal{T}$ (resp. $\mathcal{F}$ ) and the endomorphism $\mathcal{B}$ (resp. $\mathcal{C}$ ) are parallel if $\bar{\nabla} \mathcal{T} \equiv 0($ resp. $\bar{\nabla} \mathcal{F} \equiv 0)$ and $\bar{\nabla} \mathcal{B} \equiv 0$ (resp. $\bar{\nabla} \mathcal{C} \equiv 0)$, respectively.

Taking into account of $(3.7),(3.8),(3.9), 3.10)$ and $(5.1)-(5.4)$, we have the following lemma.

Lemma 5.1. Let $M$ be a quasi bi-slant submanifold of a cosymplectic manifold $\bar{M}$. Then for any $Z_{1}, Z_{2} \in \Gamma(T M)$ and $W_{1} \in \Gamma\left(T^{\perp} M\right)$ we obtain

$$
\begin{gathered}
\left(\bar{\nabla}_{Z_{1}} \mathcal{T}\right) Z_{2}=\mathcal{A}_{\mathcal{F} Z_{2}} Z_{1}+\mathcal{B} h\left(Z_{1}, Z_{2}\right) \\
\left(\bar{\nabla}_{Z_{1}} \mathcal{F}\right) Z_{2}=\mathcal{C} h\left(Z_{1}, Z_{2}\right)-h\left(Z_{1}, \mathcal{T} Z_{2}\right) \\
\left(\bar{\nabla}_{Z_{1}} \mathcal{B}\right) W_{1}=\mathcal{A}_{\mathcal{C} W_{1}} Z_{1}+\mathcal{T} A_{W_{1}} Z_{1} \\
\left(\bar{\nabla}_{Z_{1}} \mathcal{C}\right) W_{1}=-\mathcal{F} \mathcal{A}_{W_{1}} Z_{1}-h\left(Z_{1}, \mathcal{B} W_{1}\right) .
\end{gathered}
$$

First, we have the following theorem:

Theorem 5.2. Let $M$ be a quasi bi-slant submanifold of a cosymplectic manifold $\bar{M}$. Then, $\mathcal{T}$ is parallel if and only if the invariant distribution $\mathcal{D}$ is totally geodesic.

Proof. For any $X, Y \in \Gamma(\mathcal{D})$, from $(5.5)$, we have

$$
\left(\bar{\nabla}_{X} \mathcal{T}\right) Y=\mathcal{B} h(X, Y)
$$

here we have used $\mathcal{A}_{\mathcal{F} Y} X=0$ since $\mathcal{F} Y=0$ for any $Y \in \Gamma(\mathcal{D})$. Thus, our assertion comes from (5.9.

Theorem 5.3. Let $M$ be a quasi bi-slant submanifold of a cosymplectic manifold $\bar{M}$. Then if $\mathcal{F}$ is parallel if and only if

$$
g\left(\mathcal{A}_{\mathcal{C} V} Z_{2}, Z_{1}\right)=-g\left(\mathcal{A}_{V} Z_{1}, \mathcal{T} Z_{2}\right)
$$

for any $Z_{1}, Z_{2} \in \Gamma(T M)$ and $V \in \Gamma\left(T^{\perp} M\right)$. 
Proof. Assume that $F$ is parallel. Now, from (5.6), we have

$$
\left(\bar{\nabla}_{Z_{1}} \mathcal{F}\right) Z_{2}=\mathcal{C} h\left(Z_{1}, Z_{2}\right)-h\left(Z_{1}, \mathcal{T} Z_{2}\right) \text {. }
$$

Now, taking inner product with $V \in \Gamma\left(T^{\perp} M\right)$ in the above equation and using 2.5, we obtain

$$
\begin{aligned}
g\left(\left(\bar{\nabla}_{Z_{1}} \mathcal{F}\right) Z_{2}, V\right) & =g\left(\mathcal{C} h\left(Z_{1}, Z_{2}\right)-h\left(Z_{1}, \mathcal{T} Z_{2}\right), V\right) \\
& =g\left(\mathcal{C} h\left(Z_{1}, Z_{2}\right), V\right)-g\left(h\left(Z_{1}, \mathcal{T} Z_{2}\right), V\right) \\
& =-g\left(h\left(Z_{1}, Z_{2}\right), \varphi V\right)-g\left(\bar{\nabla}_{Z_{1}} \mathcal{T} Z_{2}, V\right) \\
& =-g\left(\mathcal{A}_{\mathcal{C} V} Z_{2}, Z_{1}\right)+g\left(\mathcal{T} Z_{2}, \bar{\nabla}_{Z_{1}} V\right) \\
& =-g\left(\mathcal{A}_{\mathcal{C} V} Z_{2}, Z_{1}\right)+g\left(\mathcal{T} Z_{2},-\mathcal{A}_{V} Z\right)
\end{aligned}
$$

which gives the assertion.

Theorem 5.4. Let $M$ be a quasi bi-slant submanifold of a cosymplectic manifold $\bar{M}$. Then $\mathcal{F}$ is parallel if and only if $\mathcal{B}$ is parallel.

Proof. By using (2.5), 5.6 and (5.7), we get

$$
\begin{aligned}
g\left(\left(\bar{\nabla}_{Z_{1}} \mathcal{F}\right) Z_{2}, W_{1}\right) & =g\left(\mathcal{C} h\left(Z_{1}, Z_{2}\right), W_{1}\right)-g\left(h\left(Z_{1}, \mathcal{T} Z_{2}\right), W_{1}\right) \\
& =-g\left(h\left(Z_{1}, Z_{2}\right), \mathcal{C} W_{1}\right)-g\left(\mathcal{A}_{W_{1}} Z_{1}, \mathcal{T} Z_{2}\right) \\
& =-g\left(\mathcal{A}_{\mathcal{C} W_{1}} Z_{1}, Z_{2}\right)+g\left(\mathcal{T} \mathcal{A}_{W_{1}} Z_{1}, Z_{2}\right) \\
& =-g\left(\mathcal{A}_{\mathcal{C} W_{1}} Z_{1}-\mathcal{T} \mathcal{A}_{W_{1}} Z_{1}, Z_{2}\right) \\
& =-g\left(\left(\bar{\nabla}_{Z_{1}} \mathcal{B}\right) W_{1}, Z_{2}\right)
\end{aligned}
$$

for any $Z_{1}, Z_{2} \in \Gamma(T M)$ and $W_{1} \in \Gamma\left(T^{\perp} M\right)$. This proves our assertion.

Finally, we mention another non-trivial example of quasi bi-slant submanifold of a cosymplectic manifold.

Example. Let $M$ be a submanifold of $\mathbb{R}^{11}$ defined by

$$
x(u, v, t, r, s, k, z)=\left(u, v, t, \frac{1}{\sqrt{2}} r, \frac{1}{\sqrt{2}} r, 0, s, k \cos \alpha, k \sin \alpha, 0, z\right) .
$$

We can easily to see that the tangent bundle of $M$ is spanned by the tangent vectors

$$
\begin{gathered}
e_{1}=\frac{\partial}{\partial x_{1}}, e_{2}=\frac{\partial}{\partial y_{1}}, e_{3}=\frac{\partial}{\partial x_{2}}, e_{4}=\frac{1}{\sqrt{2}} \frac{\partial}{\partial y_{2}}+\frac{1}{\sqrt{2}} \frac{\partial}{\partial x_{3}}, \\
e_{5}=\frac{\partial}{\partial x_{4}}, e_{6}=\cos \alpha \frac{\partial}{\partial y_{4}}+\sin \alpha \frac{\partial}{\partial x_{5}}, e_{7}=\frac{\partial}{\partial z}=\xi .
\end{gathered}
$$

We define the almost contact structurev $\varphi$ of $\mathbb{R}^{11}$, by

$$
\varphi\left(\frac{\partial}{\partial x_{i}}\right)=\frac{\partial}{\partial y_{i}}, \quad \varphi\left(\frac{\partial}{\partial y_{j}}\right)=-\frac{\partial}{\partial x_{j}}, \quad \varphi\left(\frac{\partial}{\partial z}\right)=0, \quad 1 \leq i, j \leq 5 .
$$


For any vector field $Z=\lambda_{i} \frac{\partial}{\partial x_{i}}+\mu_{j} \frac{\partial}{\partial y_{j}}+\nu \frac{\partial}{\partial z} \in \Gamma\left(T \mathbb{R}^{11}\right)$, then we have

$$
g(Z, Z)=\lambda_{i}^{2}+\mu_{j}^{2}+\nu^{2}, \quad g(\varphi Z, \varphi Z)=\lambda_{i}^{2}+\mu_{j}^{2}
$$

and

$$
\varphi^{2} Z=-\lambda_{i} \frac{\partial}{\partial x_{i}}-\mu_{j} \frac{\partial}{\partial y_{j}}=-Z
$$

for any $i, j=1, \ldots, 5$. It follows that $g(\varphi Z, \varphi Z)=g(Z, Z)-\eta^{2}(Z)$. Thus $(\varphi, \xi, \eta, g)$ is an is an almost contact metric structure on $\mathbb{R}^{11}$. Thus we have

$$
\begin{gathered}
\varphi e_{1}=\frac{\partial}{\partial y_{1}}, \varphi e_{2}=\frac{\partial}{\partial x_{1}}, \varphi e_{3}=\frac{\partial}{\partial y_{2}}, \varphi e_{4}=-\frac{1}{\sqrt{2}} \frac{\partial}{\partial x_{2}}+\frac{1}{\sqrt{2}} \frac{\partial}{\partial y_{3}}, \\
\varphi e_{5}=\frac{\partial}{\partial y_{4}}, \varphi e_{6}=-\cos \alpha \frac{\partial}{\partial x_{4}}+\sin \alpha \frac{\partial}{\partial y_{5}}, \varphi e_{7}=0 .
\end{gathered}
$$

By direct calculations, we obtain the distribution $\mathcal{D}=\operatorname{span}\left\{e_{1}, e_{2}\right\}$ is an invariant distribution, the distribution $\mathcal{D}_{1}=\operatorname{span}\left\{e_{3}, e_{4}\right\}$ is a slant distribution with slant angle $\theta_{1}=\frac{\pi}{4}$ and the distribution $\mathcal{D}_{2}=\operatorname{span}\left\{e_{5}, e_{6}\right\}$ is also a slant distribution with slant angle $\theta_{2}=\alpha, 0<\alpha<\frac{\pi}{2}$. Thus $M$ is a 7 -dimensional proper quasi bi-slant submanifold of $\mathbb{R}^{11}$ with its usual almost contact metric structure.

Acknowledgement. The authors are grateful to the referees for their constructive suggestions and comments.

\section{REFERENCES}

[1] Blair, D. E., Contact manifolds in Riemannian geometry, Lecture Notes in Mathematic Springer-Verlag, New York, Vol. 509, 1976.

[2] Blair, D. E., The theory of quasi-Sasakian structure, J. Differential Geom. 1, (3-4) (1967), 331-345.

[3] Carriazo, A., New developments in slant submanifolds theory, Narasa Publishing Hause New Delhi, India, 2002.

[4] Carriazo, A., Bi-slant immersions, Proceeding of the ICRAMS 2000, Kharagpur, (2000), 8897.

[5] Chen, B. Y., Geometry of slant submanifolds, Katholieke Universiteit Leuven, Leuven, Belgium, View at Zentralblatt Math., 1990.

[6] Chen, B. Y., Slant immersions, Bull. Austral. Math. Soc., 41 (1990), 135-147.

[7] Cabrerizo, J. L., Carriazo, A., Fernandez, L. M., Fernandez, M., Slant submanifolds in Sasakian manifolds, Glasgow Math. J., 42 (2000), 125-138.

[8] Cabrerizo, J. L., Carriazo, A., Fernandez, L. M., Fernandez, M., Semi-slant submanifolds of a Sasakian manifold, Geom. Dedic., 78(2) (1999), 183-199.

[9] Dirik, S., Atçeken, M., On the geometry of pseudo-slant submanifolds of a cosymplectic manifold, International Electronic Journal of Geometry, 9(1) (2016), 45-56.

[10] Dirik, S., Atceken, M., Contact pseudo-slant submanifolds of a cosymplectic manifold, New trends in Mathematical Sciences, 6(4) (2018), 154-164.

[11] Gupta, R. S., Haider, S. K., Sharfuddin, A. Slant submanifolds in cosymplectic manifolds, Colloquium Mathematicum, 105 (2016), 207-219.

[12] Khan, M. A., Totally umbilical hemi slant submanifolds of Cosymplectic manifolds, Mathematica Aeterna, 3(8) (2013), 645-653. 
A NOTE ON QUASI BI-SLANT SUBMANIFOLDS OF COSYMPLECTIC MANIFOLDS 1521

[13] Kim, U. K., On anti-invariant submanifolds of cosymplectic manifolds, Bulletin of the Korean Mathematical Society, 21(1) (1984), 35-37.

[14] Lone, M. A., Lone, M. S., Shahid, M. H., Hemi-slant submanifolds of cosymplectic manifolds, Cogent Mathematics, 3(1) (2016),1204143.

[15] Lotta, A., Slant submanifolds in contact geometry, Bulletin Mathematical Society Roumanie, 39 (1996), 183-198.

[16] Ludden, G. D., Submanifolds of cosymplectic manifolds, Journal of Differential Geometry, 4 (1970), 237-244.

[17] Olzsak, Z., On almost cosymplectic manifolds, Kodai Math J., 4 (1981), 239-250.

[18] Uddin, S., Ozel, C., Khan, V. A., A classication of a totally umbilical slant submanifold of cosymplectic manifolds, Hindawi puplishing corporation abstract applied analysis, article ID 716967, 8 pages $(2012)$

[19] Papaghuic, N., Semi-slant submanifolds of a Kaehlarian manifold, An. St. Univ. Al. I. Cuza. Univ. Iasi, 40 (2009), 55-61.

[20] Prasad, R., Verma, S. K., Kumar, S., Quasi hemi-slant submanifolds of sasakian manifolds, Journal of Mathematical and Computational Science, 10(2) (2020), 418-435.

[21] Şahin, B., Warped product submanifolds of a Kaehler manifold with a slant factor, Annales Polonici Mathematici, 95 (2009), 107-226.

[22] Şahin, B., Slant submanifolds of an almost product Riemannian manifold, Journal of the Korean Mathematical Society, 43(4) (2006), 717-732.

[23] Şahin, B., Keleş, S. Slant submanifolds of Kaehler product manifolds, Turkish Journal of Mathematics, 31 (2007), 65-77.

[24] Taştan, H. M., Özdemir, F,. The geometry of hemi-slant submanifolds of a locally product Riemannian manifold, Turkish Journal of Mathematics, 39 (2015), 268-284. 